\title{
A Novel Ferroptosis-Related IncRNA Signature for Prognosis Prediction in Patients with Papillary Renal Cell Carcinoma
}

\author{
Ruijie Dang ${ }^{1, *}$ \\ Meiling Jin ${ }^{2, *}$ \\ Jingzhu $\mathrm{Nan}^{3}$ \\ Xuege Jiang ${ }^{4}$ \\ Zheng $\mathrm{He}^{5}$ \\ Fang $\mathrm{Su}^{6}$ \\ Diangeng $\mathrm{Li} \mathbb{( D}^{7}$
}

'Department of Oncology, The First Medical Centre, Chinese PLA General Hospital, Beijing, 100853, People's Republic of China; ${ }^{2}$ Department of Nephrology, Beijing-Chaoyang Hospital, Capital Medical University, Beijing, 100020, People's Republic of China; ${ }^{3}$ Department of Clinical Laboratory, the First Medical Centre, Chinese PLA General Hospital, Beijing, I00853, People's Republic of China; ${ }^{4}$ Respiratory Diseases Department, Second Medical Center of PLA General Hospital, Beijing, I00853, People's Republic of China; ${ }^{5}$ Department of Clinical Laboratory, Chinese PLA General Hospital, Beijing, I00853, People's Republic of China;

${ }^{6}$ Aeronautical Physiological Identification Training Laboratory, Air Force Medical Center, PLA, Beijing, I00I42, People's Republic of China; ${ }^{7}$ Department of Scientific Research, Beijing-Chaoyang Hospital, Capital Medical University, Beijing, 100020, People's Republic of China

*These authors contributed equally to this work

Correspondence: Diangeng $\mathrm{Li}$ Department of Scientific Research, Beijing-Chaoyang Hospital, Capital Medical University, Beijing, 100020,

People's Republic of China

Tel/Fax +86-10-85231049

Email lidiangeng@|26.com
Purpose: Papillary renal cell carcinoma (PRCC) is a common renal cell carcinoma. Recent studies have reported that ferroptosis is involved in the occurrence and development of tumors. Long non-coding RNAs can be used as independent biomarkers for the diagnosis and prognosis of a variety of tumors.

Methods: Gene expression profile and clinical information of patients with PRCC were obtained from The Cancer Genome Atlas (TCGA) database. Lasso penalized Cox regression and univariate Cox regression analysis were utilized for model construction. The Kaplan-Meier (K-M) and receiver operating characteristic (ROC) curves were plotted to validate the predictive effect of the prognostic signature. Immune cell infiltration and immune function were compared between the high-risk and low-risk groups. Chemotherapy sensitivity analysis was also performed.

Results: We constructed a prognostic signature consisting of 15 ferroptosis-related IncRNAs. The K-M curves validated the fine predictive accuracy of the prognostic signature $(p<0.001)$. The area under the curve (AUC) of the lncRNA signature was 0.930 , exhibiting robust prognostic capacity. The high-risk group had a greater degree of immune cell infiltration than the low-risk group. Significant differences in inflammation promotion, parainflammation, and type I IFN response were noted between the low-risk and high-risk groups $(p<0.01)$. The expression levels of immune checkpoints including CD80, IDO1, and LAG3 were significantly higher in the high-risk group than in the low-risk group $(p<0.05)$. Chemotherapy sensitivity analysis showed that MNX1-AS1, ZFAS1, MIR4435-2HG, and ADAMTS9-AS1 were significantly correlated with the sensitivity of some chemotherapy drugs $(p<0.05)$.

Conclusion: We demonstrated that a ferroptosis-related lncRNA prognostic signature could be a novel biomarker for PRCC.

Keywords: ferroptosis, long non-coding RNA, PRCC, prognostic signature

\section{Introduction}

Renal cell carcinoma (RCC) is one of the common malignancies originating from the renal tubular epithelium, with an incidence of approximately 431,288 in 2020, accounting for $2.2 \%$ of common malignancies worldwide. ${ }^{1}$ Renal papillary cell carcinoma (PRCC) is the second most common renal cell carcinoma after renal clear cell carcinoma, accounting for $15 \%-20 \%$ of RCC cases. ${ }^{2}$ On the basis of different clinicopathological and immunohistochemical features, PRCC can be divided into two subtypes: type 1 and type 2; in general, type 2 PRCC has 
a worse prognosis than type 1 PRCC. $^{3}$ Although the comprehensive treatment of PRCC has improved, the overall survival (OS) rate remains low, especially for patients with advanced or metastatic tumors with limited treatment options. ${ }^{4}$ Therefore, new treatment targets and prognostic biomarkers for PRCC are necessary.

Unlike cellular autophagy and apoptosis, ferroptosis is an oxidative, iron-dependent form of necrotic cell death that is driven by the lethal accumulation of lipid peroxidation. Cancer cells have an increased iron requirement compared with normal non-cancerous cells, and studies have found that increased iron-dependent ROS-induced extra-mitochondrial lipid peroxidation triggers ferroptosis to inhibit tumorigenesis, making ferroptosis inducers a potential therapeutic agent for tumors. ${ }^{5,6}$ Ferroptosisrelated genes can be divided into three groups: driver, suppressor, and marker genes. ${ }^{7}$ Long non-coding RNAs (lncRNAs) are a subset of non-coding RNA molecules of about $200 \mathrm{nt}$, and studies have shown that lncRNAs play important roles in many life processes such as epigenetic regulation, cell cycle regulation, and cell differentiation regulation. $^{8-10}$ LncRNAs are involved in tumorigenesis, development, and metastasis processes. ${ }^{11,12}$ Currently, studies on iron death-related lncRNAs are limited. Lu et al reported that lncRNA PVT1 regulates ferroptosis through miR-214-mediated TFR1 and p53. ${ }^{13}$ LncRNA LINC00336 suppresses ferroptosis in lung cancer by acting as a competitive endogenous RNA. $^{14}$ The relationship between ferroptosis-related lncRNA and clinicopathological features of PRCC is unclear.

The Cancer Genome Atlas (TCGA) is one of the most ambitious and successful cancer genomics programs to date. The TCGA program has generated, analyzed, and provided available genomic sequence, expression, methylation, and copy number variation data on over 11,000 individuals who represent over 30 different types of cancer. ${ }^{15}$ In this study, we constructed the prognostic signature of differentially expressed ferroptosis-related IncRNAs in PRCC based on TCGA data. We confirmed that this lncRNA signature could be used as an effective and independent prognostic biomarker in patients with PRCC.

\section{Materials and Methods}

\section{Data Collection and Preprocessing}

The RNA-seq transcriptome data and clinical data of patients with PRCC were downloaded from TCGA database (https:// portal.gdc.cancer.gov/). A total of 32 adjacent normal tissues and 289 cancer tissues from patients with PRCC were enrolled. The ferroptosis-related genes were downloaded from FerrDb (http://www.zhounan.org/ferrdb/) ${ }^{16}$, and we identified 259 ferroptosis-related genes as shown in Table 1.

\section{Identification and Differential Expression Analysis of Ferroptosis-Related IncRNAs}

Pearson correlation analysis between the ferroptosisrelated genes and lncRNAs was used to identify the ferroptosis-related lncRNAs $(|\mathrm{R}|>0.5$ and $p<0.001)$. The Wilcoxon signed-rank test was utilized to identify differentially expressed ferroptosis-related genes and differentially expressed ferroptosis-related lncRNAs by the limma package of $R$ based on the cutoff values: $|\log 2 \mathrm{FC}|>1$ and FDR $<0.05$.

The "clusterProfiler" package was used to perform Gene Ontology (GO) and Kyoto Encyclopedia of Genes and Genomes (KEGG) analyses on differentially expressed ferroptosis-related genes. ${ }^{17,18}$

\section{Development of the Ferroptosis-Related IncRNA Prognostic Signature}

Lasso penalized Cox regression and univariate Cox regression analysis were employed to construct the ferroptosis-related IncRNA prognostic signature, which was used to construct a risk scoring function. The following formula was used to calculate the riskScore with the constructed risk model for all clinical cases: RiskScore $=($ coefficient $\operatorname{lncRNA} 1 \times$ expression of lncRNA1) + (coefficient 1 ncRNA2 $\times$ expression of lncRNA2) $+\cdots+$ (coefficient lncRNAn $\times$ expression lncRNAn). The patients were classified in either lowrisk $(<$ median number) or high-risk $(\geq$ median number $)$ group based on the median score.

The Kaplan-Meier (K-M) survival curve described the predictive power of indicators using the "survival" and "survminer" packages. The area under the curve (AUC) of the receiver operating characteristic (ROC) curve and C-index were applied to verify the predictive accuracy using the "timeROC" and "rms" packages.

\section{Building a Predictive Nomogram}

On the basis of all independently predictive variables, we constructed a prediction nomogram using the "rms" and "foreign" packages for the prediction of 1, 3, and 5-year OS of patients with PRCC. 
Table I Ferroptosis-Related Genes from Downloaded from FerrDb

\begin{tabular}{|c|c|c|}
\hline Data Set & $\begin{array}{l}\text { Gene } \\
\text { Count }\end{array}$ & Gene List \\
\hline Driver & 150 & $\begin{array}{l}\text { RPL8, IREB2, ATP5MC3, CS, EMC2, ACSF2, NOXI, CYBB, NOX3, NOX4, NOX5, DUOXI, DUOX2, G6PD, PGD, VDAC2, PIK3CA, } \\
\text { FLT3, SCP2, TP53, ACSL4, LPCAT3, NRAS, KRAS, HRAS, TF, TFRC, TFR2, SLC38AI, SLCIA5, GLS2, GOTI, CARSI, TP53, ALOX5, } \\
\text { KEAPI, HMOXI, TP53, TP53, GLS2, ATG5, ATG7, NCOA4, TF, ALOX5, ALOXI2, ALOXI2B, ALOXI5, ALOXI5B, ALOXE3, PHKG2, } \\
\text { TFRC, ACOI, IREB2, SLC38AI, GLS2, G6PDX, ULKI, ATG3, ATG4D, ATG5, BECNI, MAPILC3A, GABARAPL2, GABARAPLI, } \\
\text { ATGI6LI, WIPII, WIPI2, SNX4, ATGI3, ULK2, NCOA4, ACSL4, TP53, SATI, ALOXI5, ACSL4, LPCAT3, ALOXI5, ACSL4, KEAPI, } \\
\text { EGFR, NOX4, MAPK3, MAPKI, BID, ACSL4, ZEBI, KEAPI, DPP4, ALOXI5, ALOXI2, CDKN2A, PEBPI, SOCSI, CDOI, MYB, HMOXI, } \\
\text { MAPK8, MAPK9, MAPKI, MAPK3, SLCIA5, CHACI, MAPKI4, LINC00472, NOX4, GOTI, BECNI, PRKAA2, PRKAAI, ELAVLI, BAPI, } \\
\text { TP53, ABCCI, ACSL4, MIR6852, ACVRIB, TGFBRI, BAPI, EPASI, HILPDA, HIFIA, ALOXI2, ACSL4, HMOXI, IFNG, ANO6, LPINI, } \\
\text { HMGBI, TNFAIP3, TLR4, NOX4, ATF3, ATM, YYIAPI, EGLN2, MIOX, TAZ, MTDH, IDHI, SIRTI, TAZ, BECNI, FBXW7, PANXI, } \\
\text { DNAJB6, BACHI, ACSL4, LONPI }\end{array}$ \\
\hline Suppressor & 109 & $\begin{array}{l}\text { SLC7AII, GPX4, AKRICI, AKRIC2, AKRIC3, GPX4, RBI, HSPBI, HSFI, SLC7AII, GPX4, GCLC, SLC7AII, NFE2L2, SQSTMI, } \\
\text { NQOI, HMOXI, FTHI, MUCI, SLC3A2, MTIG, NFE2L2, SLC40AI, SLC7AII, GPX4, SLC7AII, CISDI, SLC7AII, FANCD2, GPX4, } \\
\text { NFE2L2, FTMT, HSPA5, ATF4, SLC7AII, GPX4, GPX4, HMOXI, ATF4, NFE2L2, TP53, SLC7AII, HELLS, SCD, FADS2, SRC, STAT3, } \\
\text { NFE2L2, PML, MTOR, NFSI, TP63, SLC7AII, TP53, CDKNIA, MIRI37, SLC40AI, GPX4, GPX4, ENPP2, VDAC2, FH, CISD2, SLC40AI, } \\
\text { MIR9-I, MIR9-2, MIR9-3, CBS, NFE2L2, SQSTMI, GPX4, ISCU, FTHI, ACSL3, OTUBI, CD44, LINC00336, STAT3, BRD4, PRDX6, MIRI7, } \\
\text { SCD, SESN2, NF2, ARNTL, HIFIA, JUN, CA9, HSPA5, TMBIM4, HSPA5, PLIN2, MIR2I2, FerIHCH, AIFM2, AIFM2, LAMP2, ZFP36, GPX4, } \\
\text { PROM2, CHMP5, CHMP6, AKRICI, AKRIC2, AKRIC3, CBS, NFE2L2, CAVI, GCHI }\end{array}$ \\
\hline Marker & 123 & $\begin{array}{l}\text { PTGS2, DUSPI, NOS2, NCF2, MT3, UBC, ALB, TXNRDI, SRXNI, GPX2, BNIP3, OXSRI, SELENOS, ANGPTL7, CHACI, SLC7AII, } \\
\text { DDIT4, LOC28456I, ASNS, TSC22D3, DDIT3, JDP2, SESN2, SLCIA4, PCK2, TXNIP, VLDLR, GPT2, PSATI, LURAPIL, SLC7A5, } \\
\text { HERPUDI, XBPI, ATF3, SLC3A2, CBS, ATF4, ZNF4I9, KLHL24, TRIB3, ZFP69B, ATP6VIG2, VEGFA, GDFI5, TUBEI, ARRDC3, CEBPG, } \\
\text { SNORAI6A, RGS4, BLOCIS5-TXNDC5, LOC390705, EIF2SI, KIM-I, IL6, CXCL2, RELA, HSDI7BII, AGPAT3, SETDIB, HMOXI, TF, } \\
\text { FTL, RPL8, ATP5MC3, TFRC, MAFG, IL33, FTHI, SLC40AI, TF, TFRC, FTHI, GPX4, HAMP, HSPBI, NFE2L2, STEAP3, DRD5, GPX4, } \\
\text { DRD4, MAP3K5, MAPKI4, SLC2AI, SLC2A3, SLC2A6, SLC2A8, SLC2AI2, GLUTI3, SLC2AI4, EIF2AK4, EIF2SI, ATF4, ALOX5, } \\
\text { ALOXI2, ALOXI5, ALOX5, ACSF2, IREB2, GPX4, HMGBI, HMOXI, NFE2L2, ELAVLI, SLC3A2, SLC7AII, TFAP2C, SPI, HBAI, NNMT, } \\
\text { PLIN4, HICI, STMNI, RRM2, CAPG, HNF4A, NGB, YWHAE, GABPBI, AURKA, MIR47I5, RIPKI, PRDXI, MIR30B }\end{array}$ \\
\hline
\end{tabular}

\section{Identification of the Relationship Between the Risk Score and the Clinicopathological Characteristics}

To better understand the role of risk score in the clinical outcome of PRCC, we systematically investigated the relationship between risk score and clinicopathological characteristics (including age, sex, stage status, T status, $\mathrm{M}$ status, and $\mathrm{N}$ status), as well as the expression level of prognostic lncRNAs, using the "pheatmap" package.

\section{Protein-Protein Interaction Network Construction}

The String database (https://cn.string-db.org/) was used to analyze the interactions of ferroptosis-related genes and ferroptosis-related lncRNAs. Cytoscape ${ }^{19}$ was used for the visualization of the protein-protein interaction (PPI) network.

\section{Gene Set Enrichment Analyses}

Gene set enrichment analysis (GSEA) ${ }^{20}$ was performed to explore the potential altered pathways between the highand low-risk groups.
Immunity Analysis

The CIBERSORT, ${ }^{20,21}$ ESTIMATE, ${ }^{22}$ MCPcounter, $^{23}$ single-sample gene set enrichment analysis (ssGSEA), ${ }^{24}$ and TIMER $^{25}$ algorithms were used to assess cellular components or cell immune responses. A total of 22 immune cell types were used to estimate relative abundance of immune cell infiltration between the low- and high-risk groups. ssGSEA was then used to quantify tumor-infiltrating immune cell subgroups during immune responses and immune functions between the two groups. We also compared the expression levels of immune checkpoints between the two groups.

\section{Chemotherapy Sensitivity Analysis}

We obtained the drug sensitivity data and RNA-seq data of the NCI-60 cell line from CellMiner (https://discover.nci. nih.gov/cellminer/home.do) and analyzed the relationship between the prognostic lncRNAs and drug sensitivity using Pearson correlation analysis.

\section{Statistical Analysis}

$\mathrm{R}$ version 4.0.2 software and its resource packages were used for statistical analysis and related visualization 
graphics. Student's $t$ test was used to compare differences between different risk groups. Statistical significance was set at $p<0.05$.

\section{Results}

\section{Enrichment Analysis of Ferroptosis-Related Genes}

The work flowchart is shown in Figure 1. The detailed clinical characteristics of these patients are shown in Table 2. We identified 56 differentially expressed ferroptosis-related genes (32 upregulated and 24 downregulated, Table S1). Differentially expressed genes (DEGs) were enriched in the biological processes with response to oxidative stress, cellular response to chemical stress, and cellular response to oxidative stress. They were enriched in molecular functions with oxidoreductase activity, acting on $\mathrm{NAD}(\mathrm{P}) \mathrm{H}$, oxidoreductase activity, acting on paired donors, with incorporation or reduction of molecular oxygen, and iron ion binding. Cellular component (CC) was mainly enriched in apical parts of the cell, oxidoreductase complex, and NADPH oxidase complex. The biological pathways in KEGG were enriched in arachidonic acid metabolism, biosynthesis of amino acids, leishmaniasis, HIF-1 signaling pathway, and carbon metabolism (Figure 2).

\section{Ferroptosis-Related IncRNAs' Prognosis Signature in PRCC}

A total of 680 ferroptosis-related lncRNAs were identified (Table S2). Univariate Cox analysis was performed to evaluated the relationship between the expression of ferroptosis-
Table 2 The Clinical Characteristics of Patients with PRCC in the TCGA Dataset

\begin{tabular}{|l|l|}
\hline Clinical Characteristics & TCGA Cohort (N=289) \\
\hline $\begin{array}{l}\text { Gender } \\
\text { Male/Female }\end{array}$ & $76 / 213$ \\
\hline $\begin{array}{l}\text { Age } \\
\leq 65 />65 / N A\end{array}$ & $177 / 109 / 3$ \\
\hline $\begin{array}{l}\text { Stage } \\
\text { I/II/III/V/NA }\end{array}$ & $173 / 21 / 51 / 15 / 29$ \\
\hline $\begin{array}{l}\text { M } \\
\text { MO/MI/Mx/NA }\end{array}$ & $95 / 9 / 171 / 14$ \\
\hline $\begin{array}{l}\text { N } \\
\text { No/NI/N2/Nx/NA }\end{array}$ & $49 / 24 / 4 / 211 / 1$ \\
\hline
\end{tabular}

related lncRNAs and patients' OS, and the results showed that 29 ferroptosis-related IncRNAs (DARS-AS1, AL355512.1, ZFAS1, LUCAT1, AL022344.1, LINC01559, AC010624.2, AL031710.1, AL355102.4, MNX1-AS1, AC109460.1, AC127537.1, CD44-AS1, AC099850.4, LINC02154, AC024022.1, AC092115.4, AC026401.3, CYTOR, AL365181.3, LINC02535, ADAMTS9-AS1, AC022075.1, AC096642.1, AC107464.2, SLC25A5-AS1, AC104564.1, ITGB1-DT, and MIR4435-2HG) were independent prognostic predictors of PRCC (Figure 3). Multivariate Cox analysis indicated that 15 of 29 ferroptosis-related IncRNAs were the best candidates to construct the prognostic signature (Table 3 ). The risk score was calculated for each patient with PRCC as follows: $-0.0152169179410617 \times$ the expression levels of ZFAS1 $+(-0.814201526659232) \times$ the

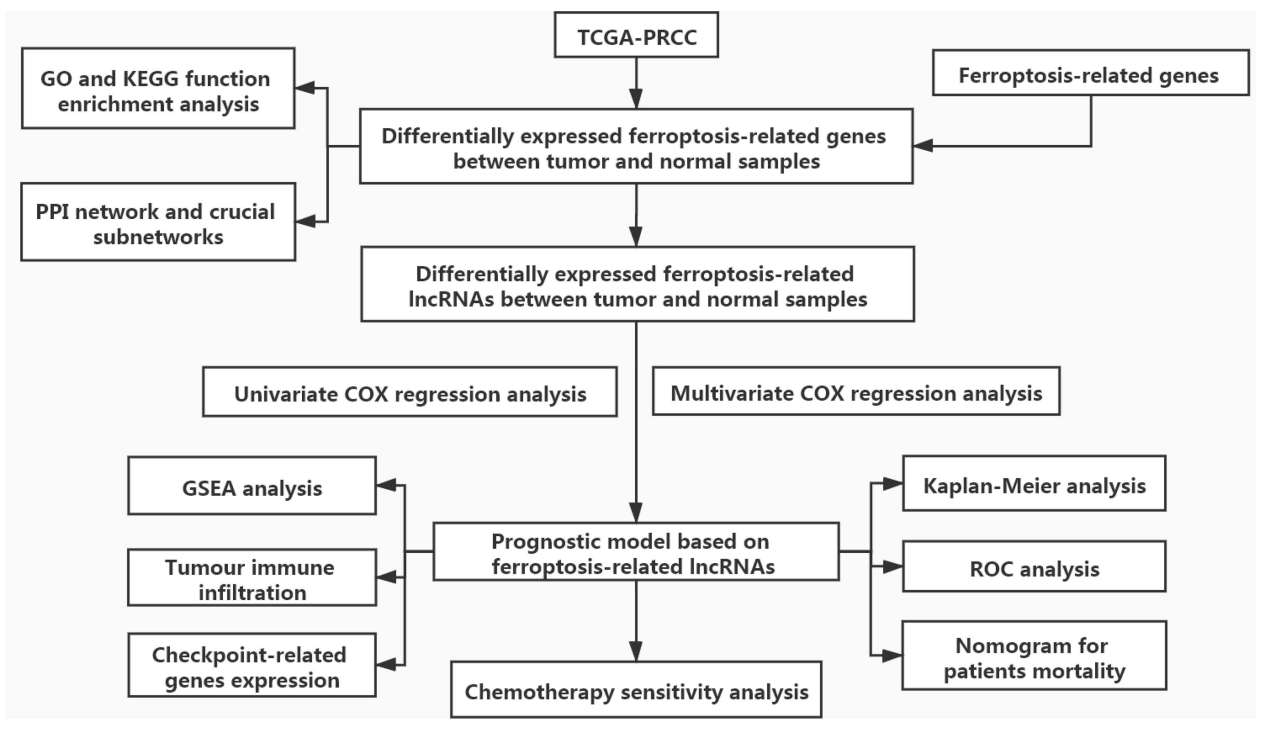

Figure I Flowchart of this study. 


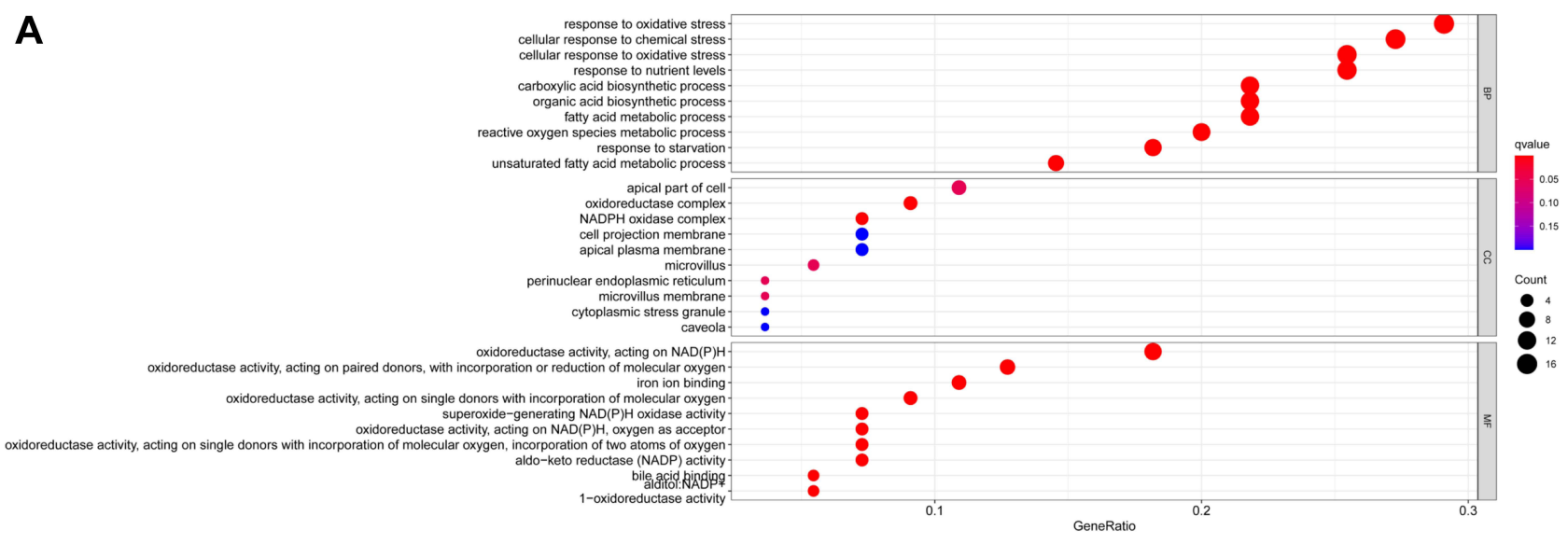

B

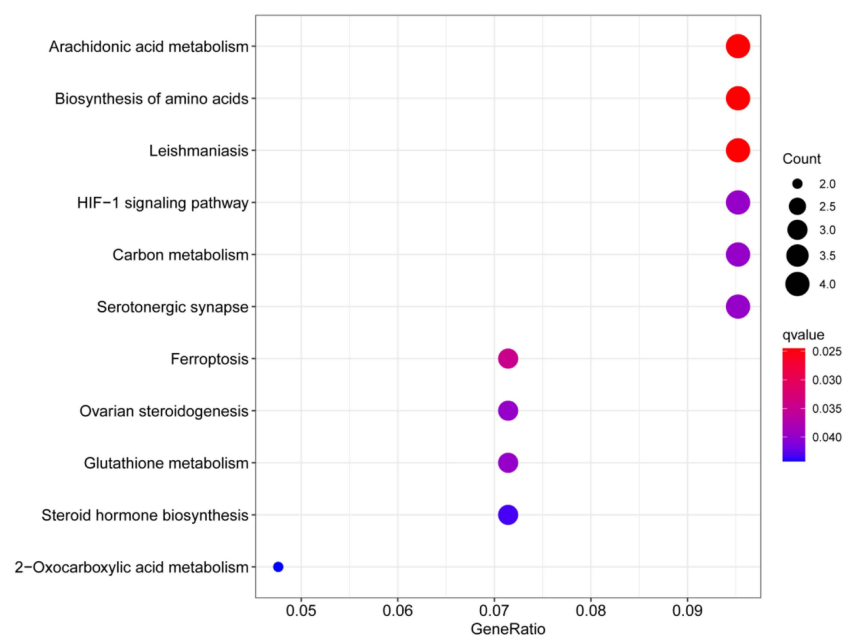

Figure 2 GO and KEGG analyses of differentially expressed ferroptosis-related genes. (A) Functional annotation of differentially expressed ferroptosis-related genes between adjacent normal and tumor samples using GO terms. (B) Functional annotation of differentially expressed ferroptosis-related genes between adjacent normal and tumor samples using KEGG terms.

expression levels of AC010624.2 + $(-0.574312213529718) \times$ the expression levels of AL031710.1 $+(-0.29571$ $1007986573) \times$ the expression levels of AL355102.4 + $0.343550090287168 \times$ the expression levels of MNX1-AS1 $+2.59391370444094 \times$ the expression levels of AC109460.1 + $0.514983257942382 \times$ the expression levels of AC127537.1+ $0.178442445321917 \times$ the expression levels of AC099850.4+ $0.0362308172908584 \times$ the expression levels of LINC02154 + $(-3.2728034303393) \times$ the expression levels of AC024022.1 + $0.142403529727197 \times$ the expression levels of AC026401.3+ $0.0664305502643028 \times$ the expression levels of LINC02535 + $(-0.0190675805173939) \times$ the expression levels of ADAMTS9-AS1 $+(-0.194060378575192) \times$ the expression levels of AC107464.2 + 0.367056382643099 $\times$ the expression levels of MIR4435-2HG. Thus, we constructed a ferroptosisrelated lncRNA prognosis model to predict the prognosis of patients with PRCC.

\section{Survival Results and Multivariate Examination}

All patients were divided into the high- and low-risk groups based on the median values of risk scores. $\mathrm{K}-\mathrm{M}$ analyses revealed that the OS of the high-risk group was significantly worse than that of the lowrisk group $(\mathrm{p}<0.001$, Figure $4 \mathrm{~A})$. The AUC was 0.930 , indicating its excellent predictive performance (Figure 4B). The AUC scores at 1, 2, and 3 years were $0.930,0.953$, and 0.933 , respectively (Figure 4C). The survival status plot showed that the patients' risk score was inversely proportional to the survival of patients with PRCC (Figure 4D and E). DCA also indicating the excellent predictive performance of the risk model (Figure 4F). The heatmap demonstrated that 10 IncRNAs were positively correlated with the risk model (Figure 4G). 
Table 3 Fifteen Prognostic Ferroptosis-Related IncRNAs Identified by Multivariate Cox Analysis

\begin{tabular}{|l|c|c|c|c|c|}
\hline LncRNA & Coefficient & HR & HR.95L & HR.95H & p-value \\
\hline ZFASI & -0.01522 & 0.984898 & 0.966031 & 1.004134 & 0.123083 \\
AC0I0624.2 & -0.8142 & 0.442993 & 0.15713 & 1.248922 & 0.123649 \\
AL03I7I0.I & -0.57431 & 0.563092 & 0.361271 & 0.87766 & 0.011204 \\
AL355I02.4 & -0.2957 I & 0.744002 & 0.539788 & 1.025475 & 0.07087 I \\
'MNXI-ASI' & 0.34355 & 1.409944 & 0.92333 & 2.153015 & 0.111692 \\
ACI09460.I & 2.593914 & 13.38204 & 3.955071 & 45.27835 & $3.03 E-05$ \\
ACI27537.I & 0.514983 & 1.67361 & 0.944937 & 2.96419 & 0.077435 \\
AC099850.4 & 0.178442 & 1.195354 & 0.988426 & 1.445603 & 0.065779 \\
LINC02I54 & 0.036231 & 1.036895 & 1.001459 & 1.073585 & 0.041135 \\
AC024022.I & -3.2728 & 0.0379 & 0.001555 & 0.923726 & 0.044573 \\
AC02640I.3 & 0.142404 & 1.153042 & 0.977286 & 1.360406 & 0.091475 \\
LINC02535 & 0.066431 & 1.068687 & 0.98249 & 1.162445 & 0.121559 \\
'ADAMTS9-ASI' & -0.01907 & 0.981113 & 0.963374 & 0.999178 & 0.040535 \\
ACI07464.2 & -0.19406 & 0.823608 & 0.725773 & 0.934631 & 0.002632 \\
'MIR4435-2HG' & 0.367056 & 1.443479 & 1.139038 & 1.829292 & 0.002388 \\
\hline
\end{tabular}

Univariate and multivariate Cox analyses were used to test whether risk score is an independent prognostic factor for OS. The lncRNA signature (HR:1.005, 95CI: $1.002-$ 1.007) and tumor stage (HR:2.232, 95CI: 1.640-3.038) were independent prognostic factors of OS of patients with PRCC (Figure 5A and B). The hybrid nomogram incorporating clinicopathological characteristics and the novel ferroptosis-related lncRNAs' prognostic signature

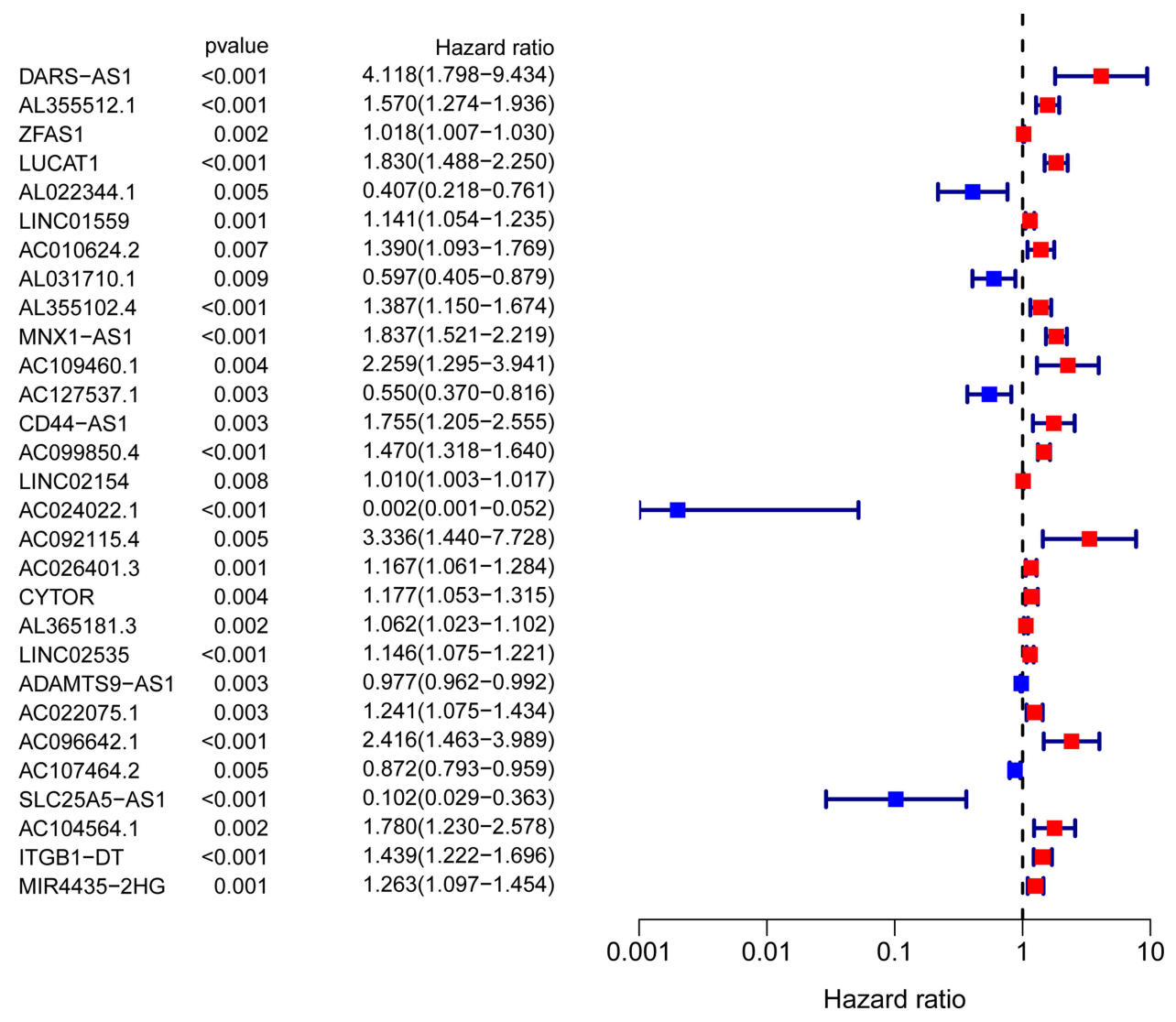

Figure 3 Univariate Cox regression analysis identified 29 ferroptosis-related IncRNAs as independent prognosis predictors of PRCC. 
A

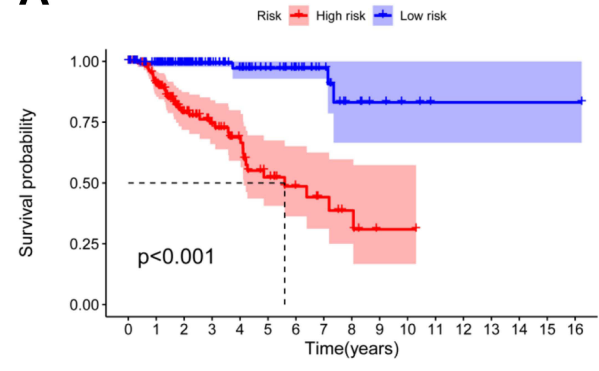

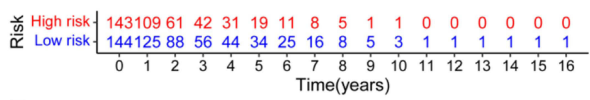

\section{D}

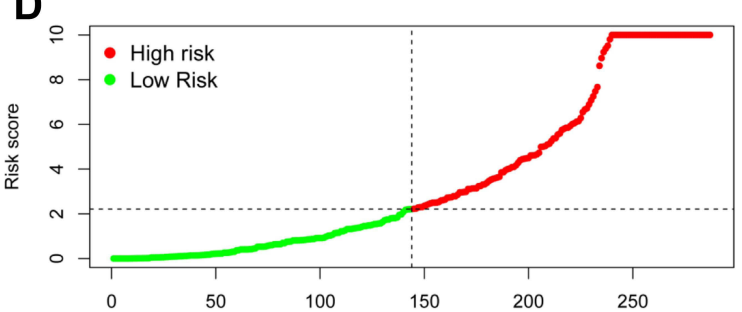

$\mathbf{E}$

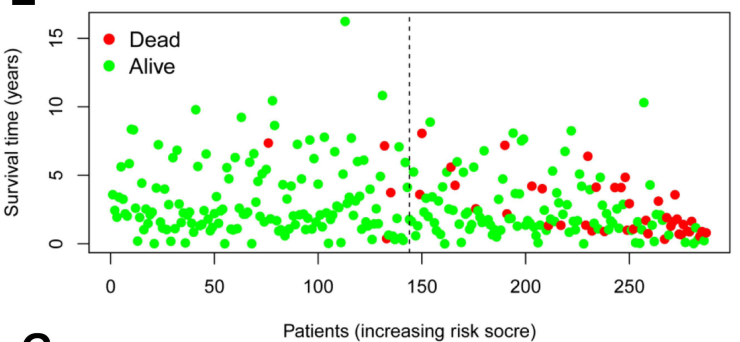

B

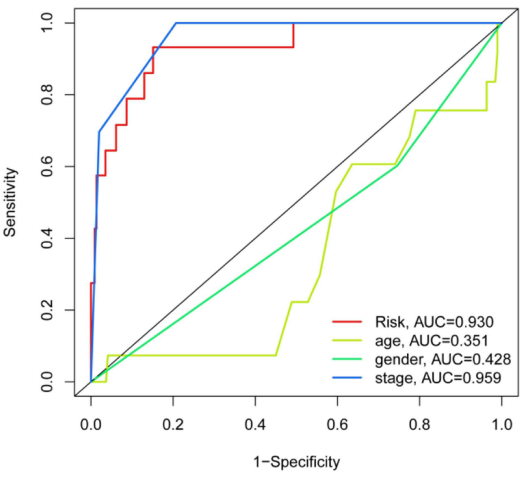

F
C

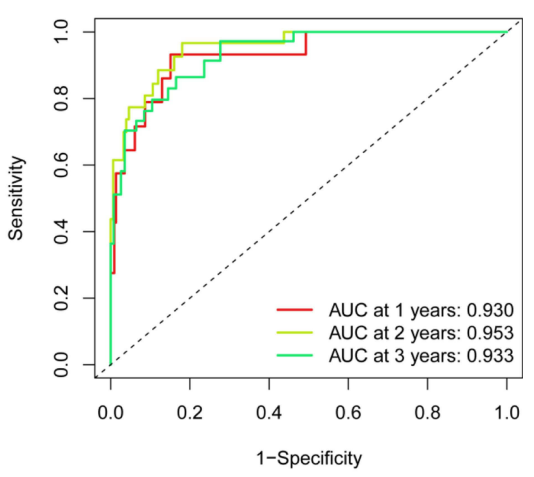

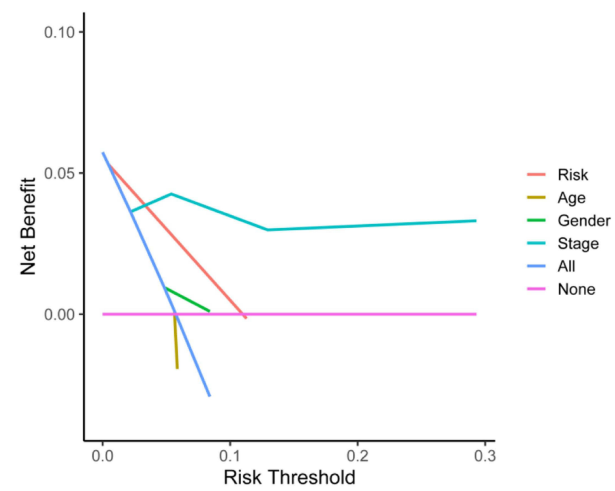

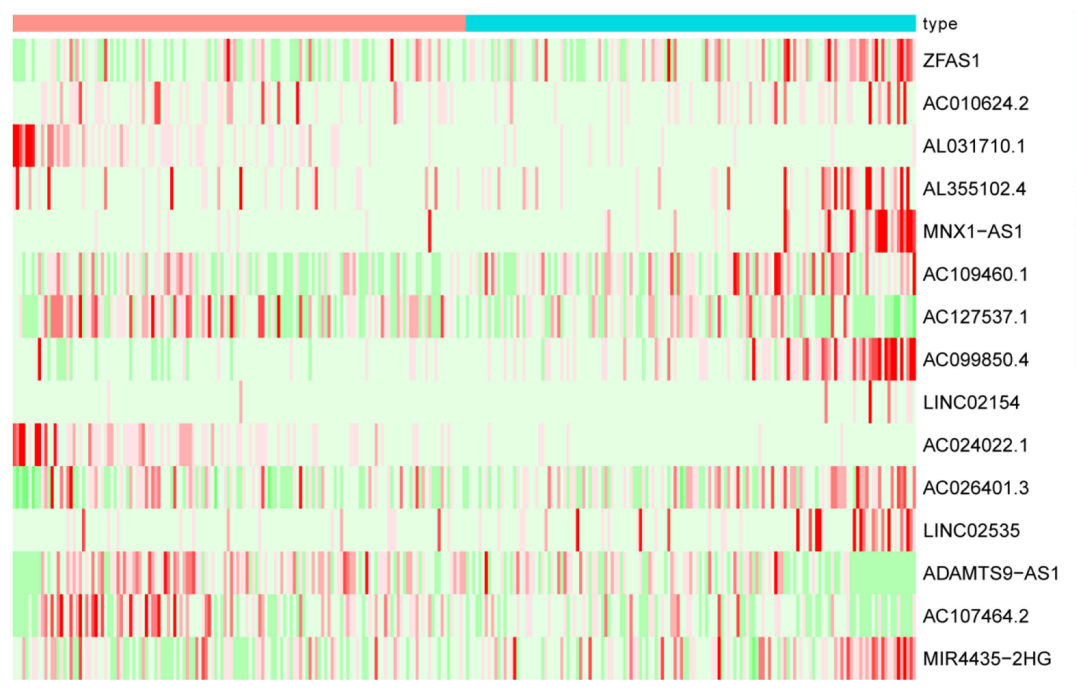

Figure 4 Ferroptosis-related IncRNAs' prognostic signature in patients with PRCC. (A) Kaplan-Meier survival curve result. (B) AUC values of the risk factors. (C) AUC values at I, 2, and 3 years (D) Distribution of risk scores of patients with high- and low-risk PRCC based on the ferroptosis-related IncRNAs' prognostic signature. (E) Scatter plot shows the correlation between the survival status of the high- and low-risk groups. (F) DCA of the risk factors. (G) Heat map of the expression of 15 ferroptosis-related IncRNAs between the high- and low-risk groups. 

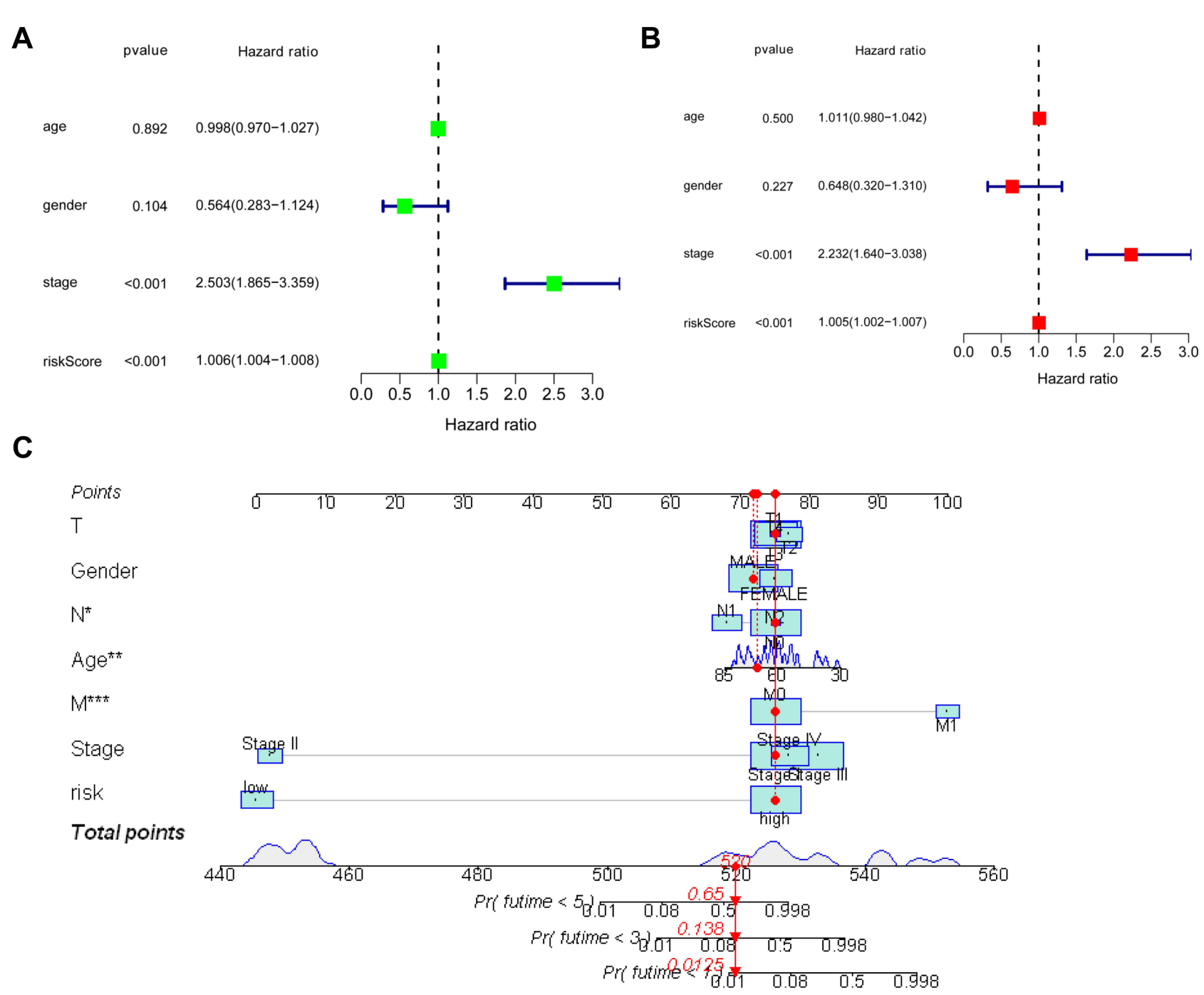

Figure 5 Univariate $(\mathbf{A})$ and multivariate $\operatorname{COX}(\mathbf{B})$ analyses between overall survival rate and various clinicopathological parameters. (C) Nomogram for both clinicopathological factors and prognostic ferroptosis-related IncRNAs $\left({ }^{*} p<0.05 ; * * p<0.01 ; * * *<<0.001\right)$.

might be applied in the clinical management of patients with PRCC (Figure 5C).

The association between ferroptosis-related lncRNAs' prognostic signature and clinicopathological manifestations was shown in the heatmap (Figure 6).

\section{Gene Set Enrichment Analysis}

The relationship between ferroptosis-related genes and lncRNAs is shown in Figure 7. GSEA revealed that the prognostic ferroptosis-related lncRNAs were associated with the tumor-related pathways and immune-related pathways, such as acute myeloid leukemia, bladder cancer, chemokine signaling pathway, primary immunodeficiency, natural killer cell-mediated cytotoxicity, and $\mathrm{T}$ cell receptor signaling pathway (Figure 8).

\section{Immune Status Difference Between Patients with High-Risk and Low-Risk PRCC}

The immune response heatmap based on CIBERSORT, ESTIMATE, MCP counter, ssGSEA, and TIMER algorithm is shown in Figure 9A. ssGSEA was used to analyze the correlation between immune cell subsets and related functions, and the results showed significant differences in inflammation promotion, 


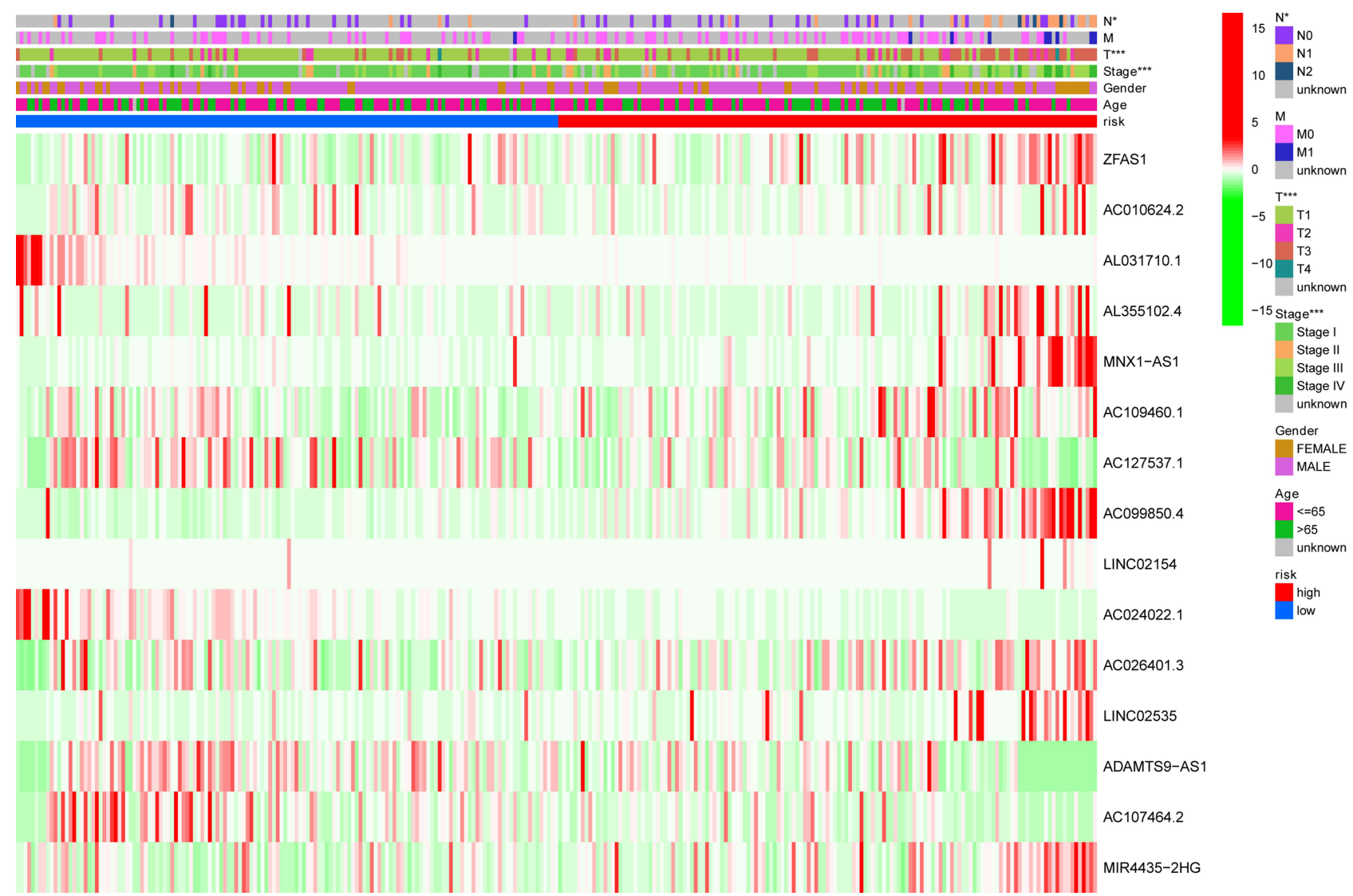

Figure 6 Heatmap for ferroptosis-related IncRNAs' prognostic signature and clinicopathological manifestations $\left(*_{p}<0.05\right.$; $* * p<0.01$; $\left.* * * p<0.00 \mathrm{I}\right)$.

parainflammation, and Type I IFN response between the low- and high-risk groups ( $p<0.01$; Figure 9B). In addition, the expression of immune checkpoints including CD80, IDO1, and LAG3 was significantly different between the low-risk and high-risk groups (Figure 9C).

\section{Prognostic IncRNA Expression and Chemotherapy Sensitivity}

We investigated the expression of prognostic lncRNAs in NCI-60 cell lines and analyzed the relationship between the expression levels and drug sensitivity. The results showed that MNX1-AS1, ZFAS1, MIR44352HG, and ADAMTS9-AS1 were significantly correlated with the sensitivity of some chemotherapy drugs (Figure 10 and Table 4). For instance, MNX1-AS1 could increase the sensitivity of most chemotherapy drugs, including fulvestrant, LDK-378, fluorouracil, tegafur, and PF-06463922. ZFAS1 could reduce the sensitivity of most chemotherapy drugs, including everolimus and rapamycin.

\section{Discussion}

Given that the molecular mechanisms of PRCC and clear cell renal cell carcinoma (RCCC) differ, and the incidence of PRCC is lower than that of PCCC, patients with PRCC are always excluded from large clinical trials of targeted drugs, and research on PRCC is not as popular as studies on RCCC. ${ }^{26}$ Although some patients with PRCC can receive early surgical treatment, a large number of advanced patients continue to die due to postoperative recurrence, tumor metastasis, or resistance to chemotherapy drugs. Therefore, new and effective prognostic biomarkers for PRCC should be explored. ${ }^{27}$ This study is the first to establish a prognostic model based on ferroptosisrelated lncRNAs in patients with PRCC.

In this study, we first identified 680 ferroptosisrelated IncRNAs, analyzed 29 prognostic ferroptosisrelated lncRNAs via multivariate Cox regression 


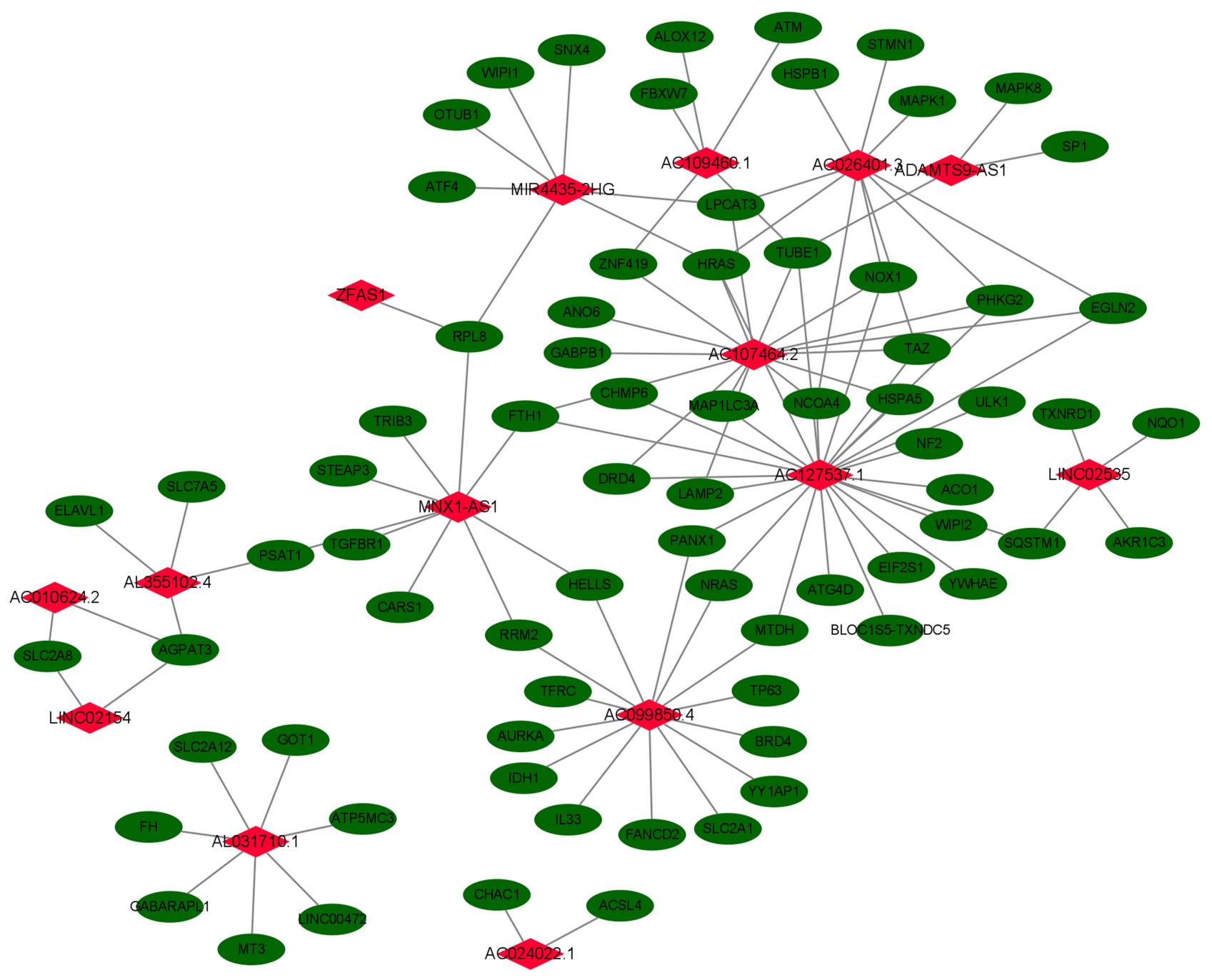

Figure 7 Relationship between the prognostic ferroptosis-related IncRNA and mRNA expression levels.

analysis, and established a ferroptosis-related lncRNA signature model for PRCC. The prognostic model of PRCC proposed in this study consisted of 15 ferroptosis-related lncRNAs. Studies have reported that these IncRNAs play important roles in the pathogenesis of tumors. ZFAS1 is a major regulator of the epithelialmesenchymal transition (EMT) through miR-200/ ZEB1/E-cadherin and vimentin signaling in colon adenocarcinoma. $^{28}$ The expression level of AL031710.1 decreases with the increase in tumor stage of papillary renal cell carcinoma. ${ }^{29}$ AL355102.4 is downregulated in clear cell renal cell carcinoma samples and indicates good prognosis when lowly expressed, which reveals that AL355102.4 is a risk factor with high gene expression. ${ }^{30}$ High MNX1-AS1 levels are correlated with poor survival outcome and aggressive clinicopathological characteristics in various cancers, suggesting that MNX1-AS1 may be applied as a prognostic marker and even a therapeutic target. ${ }^{31}$ In the miRNA-IncRNA pair centered regulatory network consisting of competing triplets in ovarian cancer, AC099850.4 is one of the top five lncRNAs with the most connections. $^{32}$ Studies have shown that LINC02154 is significantly upregulated in renal cell carcinoma, and its high expression is one of the risk factors for poor prognosis. It is involved in the construction of a model for predicting the prognosis of patients with renal cell carcinoma. ${ }^{33,34}$ AC024022.1 is a protective biomarker in papillary renal cell carcinoma. ${ }^{35}$ LINC02535 co-functions with PCBP2 to regulate DNA damage repair in cervical cancer by stabilizing RRM1 mRNA. ${ }^{36}$ LINC02535 is highly 

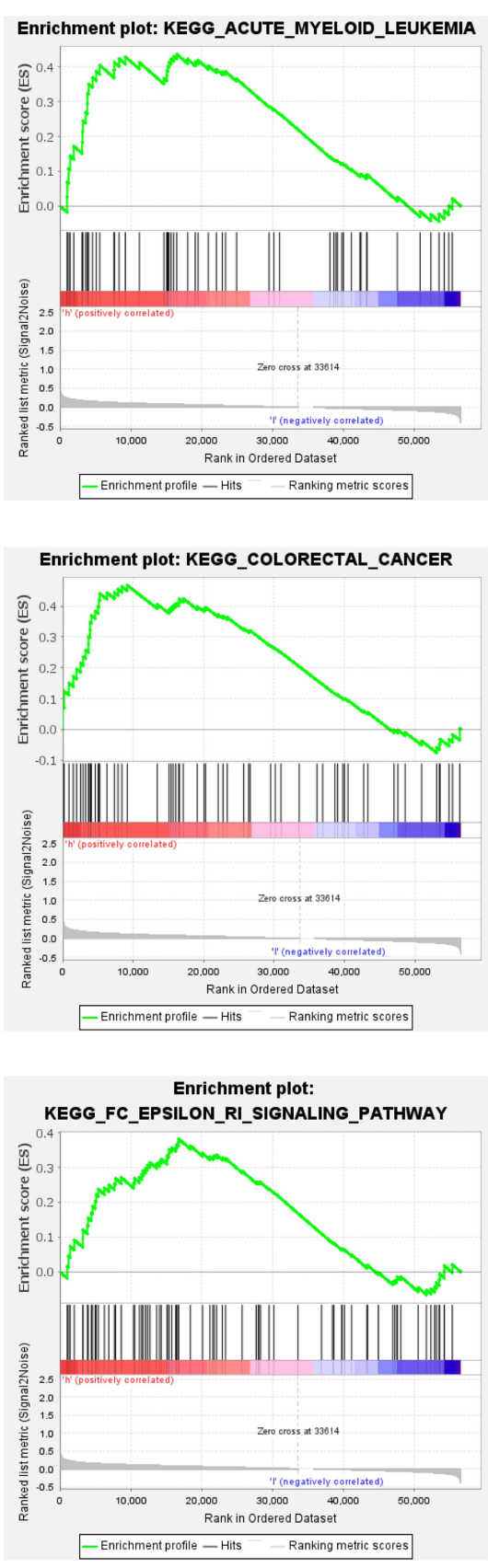
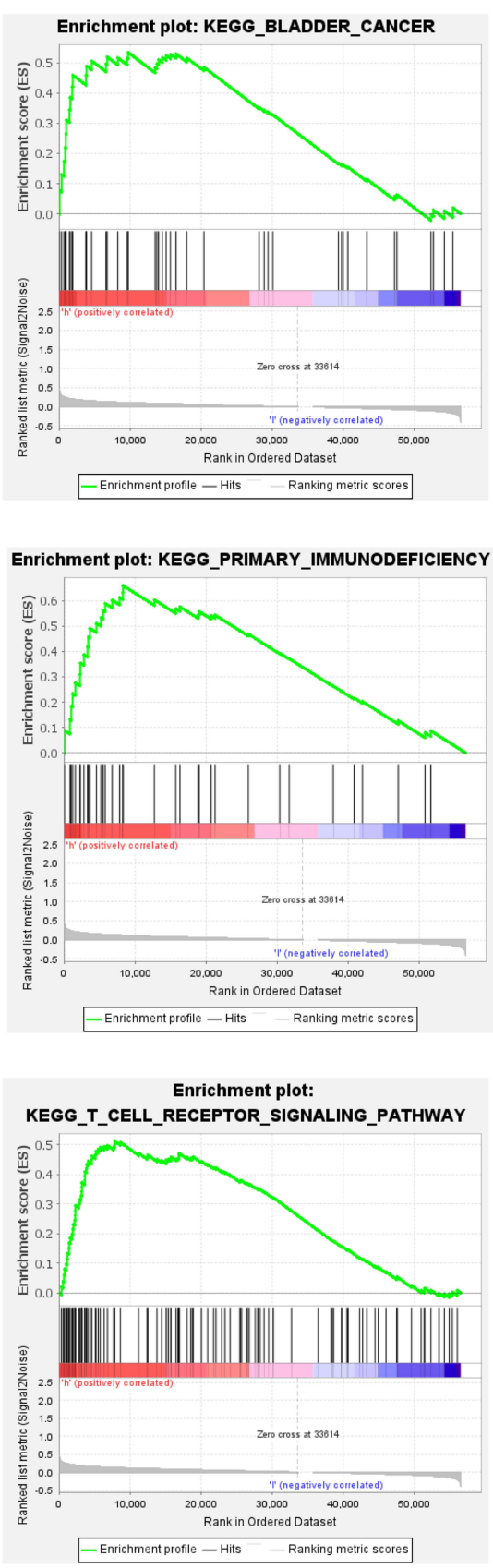
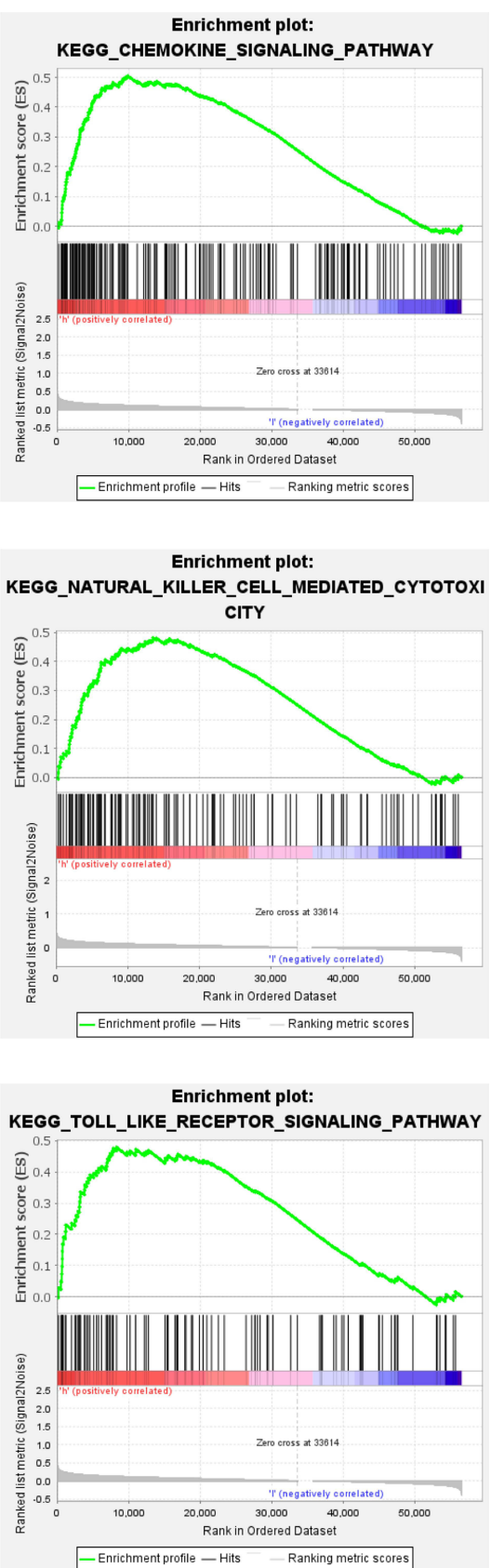

Figure 8 Gene enrichment analysis for prognostic ferroptosis-related IncRNAs.

expressed in lung Adenocarcinoma tissues and closely related to the OS of lung adenocarcinoma. Knocking down LINC02535 also significantly inhibits the EMT, a key contributor to tumor invasion and metastasis. ${ }^{37}$ ADAMTS9-AS1 restrains the aggressive traits of breast carcinoma cells via sponging miR-513a-5, ${ }^{38}$ but it promotes cell proliferation and EMT in colorectal cancer. ${ }^{39}$ MIR4435-2HG is involved in the genesis and progression of a variety of tumors. ${ }^{40-42}$ Although these lncRNAs are closely related to the pathogenesis of tumors, we combined them as prognostic markers in patients with PRCC for the first time.

Our results demonstrated that this model had good predictive performance using Kaplan-Meier and ROC curves. The results of univariate and multivariate Cox analyses suggested that this lncRNA signature and tumor stage were independent prognostic factors of OS of PRCC. The hybrid nomogram incorporating 


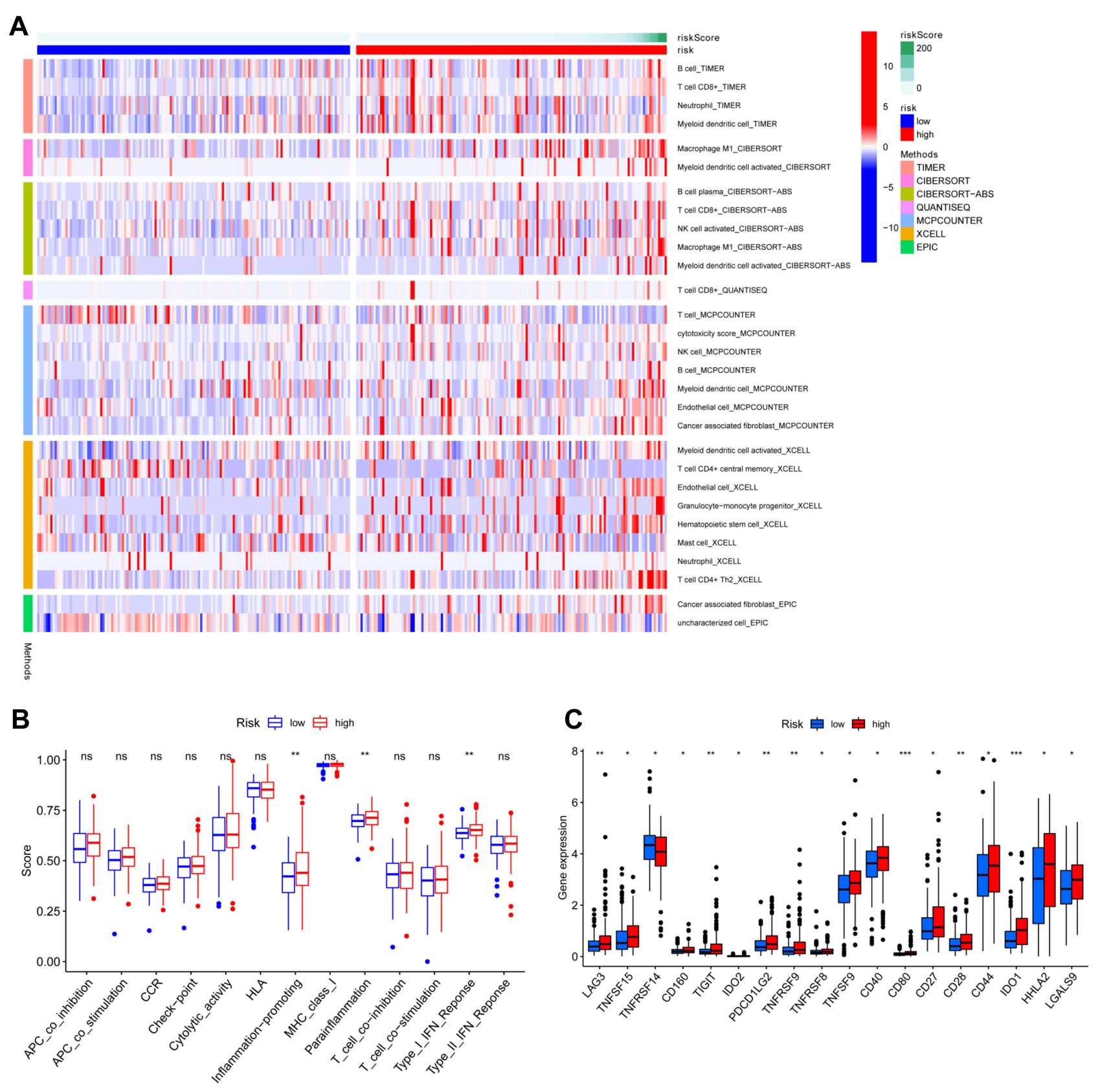

Figure 9 (A) Immune status difference between high-risk and low-risk groups. Heatmap for immune responses based on CIBERSORT, ESTIMATE, MCPcounter, ssGSEA, and TIMER algorithms among high- and low-risk groups. (B) Immune-related function between high- and low-risk groups. (C) Expression of immune checkpoints between high- and low-risk groups. $*_{p}<0.05$; $* *_{p}<0.01$; ***p $<0.001$.

clinicopathological characteristics and the novel ferroptosis-related lncRNAs' prognostic signature might be applied in the clinical management of patients with PRCC.

As ferroptosis is associated with immunity, we evaluated immune cell infiltration and immune function between the low- and high-risk groups. Compared with the low-risk group, the high-risk group had a greater degree of immune cell infiltration, such as B cells, T cell
CD8, macrophages, and $\mathrm{NK}$ cells, indicating that the high-risk group had stronger anti-tumor immune activity than the low-risk group. In addition, eeGSEA confirmed that the inflammation-related immune function in the high-risk group was more active than that in the lowrisk group. We tested the expression level of immune checkpoints and found that the expression levels of most immune checkpoints were significantly higher in the high-risk group than in the low-risk group. Given that 


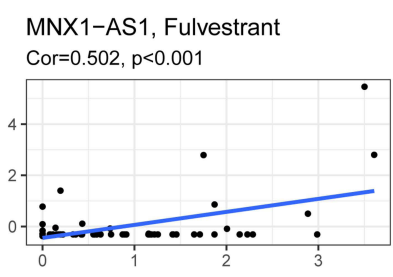

MNX1-AS1, Fluorouracil Cor $=0.405, p=0.001$
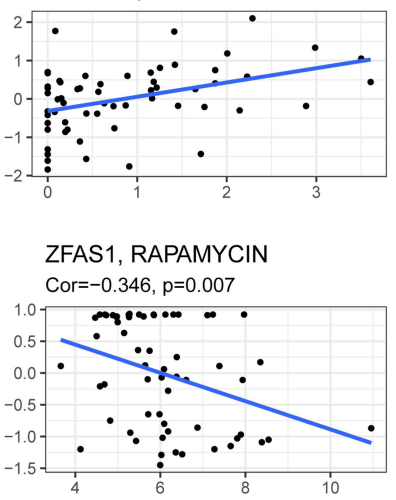

MIR4435-2HG, Ponatinib Cor $=0.329, p=0.010$

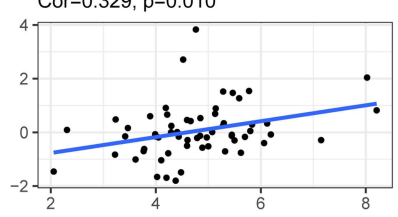

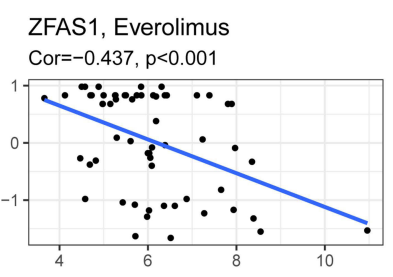
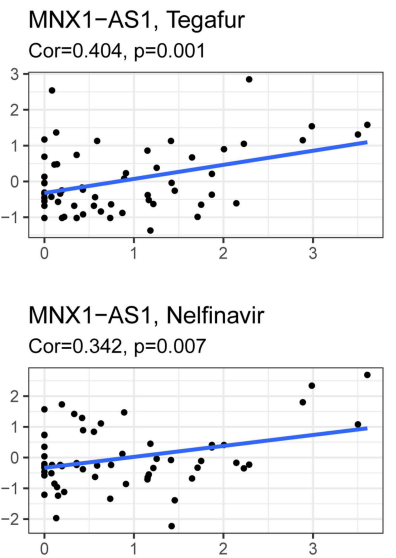

ZFAS1, Palbociclib

Cor $=0.431, p<0.001$

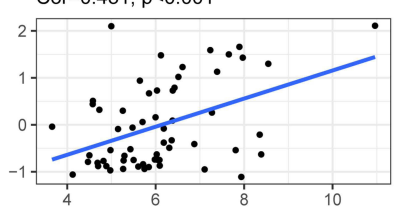

MNX1-AS1, PF-06463922

Cor $=0.373, p=0.003$

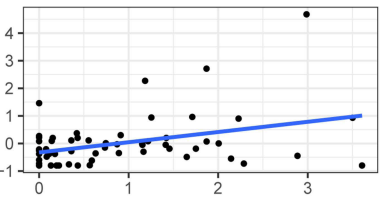

MNX1-AS1, Nitrogen mustard Cor $=0.338, p=0.008$

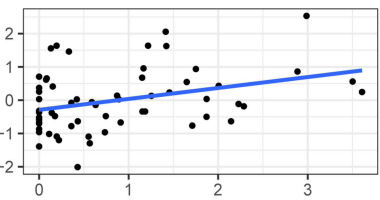

MNX1-AS1, NMS-E628

Cor $=0.328, p=0.010$

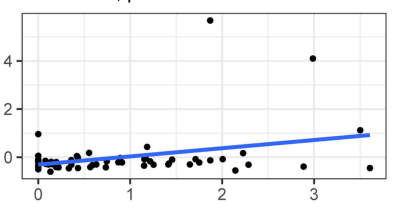

MNX1-AS1, Imiquimod Cor $=0.326, p=0.011$

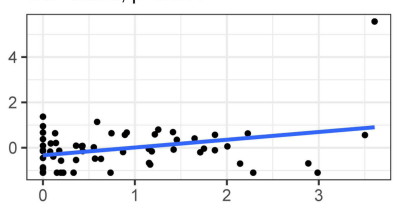

MNX1-AS1, LDK-378

Cor $=0.412, p=0.001$

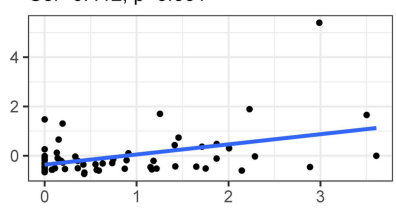

MNX1-AS1, Oxaliplatin Cor $=0.360, p=0.005$

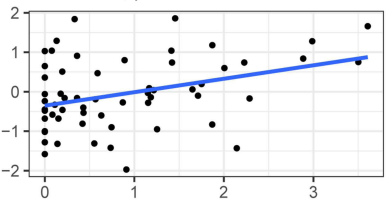

MNX1-AS1, Belinostat Cor $=0.336, p=0.009$
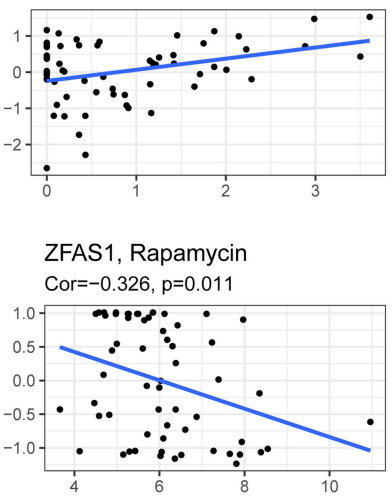

Figure 10 Prognostic IncRNA expression and chemotherapy sensitivity.

the expression of immune checkpoints is usually positively correlated with immunotherapy responsiveness, the high-risk group might better respond to immunotherapy than the low-risk group.

On the basis of the results of the chemotherapy drug sensitivity analysis, we found that the expression of some prognostic lncRNAs was related to the resistance or sensitivity of many Food and Drug Administration-approved chemotherapy drugs. The increased expression of MNX1-AS1 was associated with increased drug sensitivity. ADAMTS9-AS1 was negatively correlated with the sensitivity of Axitinib but positively correlated with sensitivity of Bosutinib, Erlotinib, Lapatinib, and Neratinib. Therefore, some prognostic lncRNAs can be used as therapeutic targets to overcome chemotherapy drug resistance or adjuvant chemotherapy drug sensitivity.
This study still had some limitations. We performed retrospective analysis using data from a public database, so bias was inevitable. Therefore, large prospective studies are needed to confirm the validity of the prognostic features.

\section{Conclusion}

We constructed and validated a ferroptosis-related lncRNA prognosis signature in PRCC, which consisted of 15 lncRNAs. This novel ferroptosis-related lncRNA prognosis signature could predict the survival of patients with PRCC. Besides, we found significant differences in immune cell infiltration and immune-related function between the highand low-risk groups, which might help for follow-up immune intervention therapy. Thus, our study might provide a theoretical basis for promising biomarkers and targets for the individualized therapy of PRCC. 
Table 4 Prognostic IncRNAs Expression and Chemotherapy Sensitivity

\begin{tabular}{|c|c|c|c|}
\hline IncRNA & Drug & Correlation Coefficient & p-value \\
\hline ADAMTS9-ASI & Erlotinib & $0.29749 \mid 402$ & 0.020975912 \\
\hline ADAMTS9-ASI & Bisacodyl, active ingredient of Viraplex & -0.283459087 & 0.028184654 \\
\hline ADAMTS9-ASI & Neratinib & 0.271310903 & 0.036005728 \\
\hline ADAMTS9-ASI & Bosutinib & 0.26315963 & 0.04220366 \\
\hline ADAMTS9-ASI & Lapatinib & 0.261745237 & 0.043363773 \\
\hline ADAMTS9-ASI & Axitinib & -0.26050053 & 0.044406292 \\
\hline ADAMTS9-ASI & STREPTOZOCIN & 0.255232655 & 0.049048955 \\
\hline MIR4435-2HG & Ponatinib & 0.328865432 & 0.010300062 \\
\hline MIR4435-2HG & Fluorouracil & -0.32535973 & 0.011192213 \\
\hline MIR4435-2HG & Simvastatin & 0.316383621 & 0.013786944 \\
\hline MIR4435-2HG & Raloxifene & -0.31546008 & 0.014081146 \\
\hline MIR4435-2HG & Tamoxifen & -0.303981248 & 0.018212357 \\
\hline MIR4435-2HG & Lenvatinib & 0.297621875 & 0.020917034 \\
\hline MIR4435-2HG & Ixabepilone & -0.293020355 & 0.023080275 \\
\hline MIR4435-2HG & Tegafur & -0.292130537 & 0.023519712 \\
\hline MIR4435-2HG & VINORELBINE & -0.286826995 & 0.026288093 \\
\hline MIR4435-2HG & Fulvestrant & -0.273018622 & 0.034808023 \\
\hline MIR4435-2HG & Dasatinib & 0.269980422 & 0.036962397 \\
\hline MIR4435-2HG & Homoharringtonine & -0.257839243 & 0.046704445 \\
\hline MNXI-ASI & Fulvestrant & $0.50187 \mid 245$ & 4.40E-05 \\
\hline MNXI-ASI & LDK-378 & 0.412043043 & 0.001071054 \\
\hline MNXI-ASI & Fluorouracil & $0.405 \mathrm{II} 6693$ & 0.001323304 \\
\hline MNXI-ASI & Tegafur & 0.404012298 & 0.001368123 \\
\hline MNXI-ASI & PF-06463922 & 0.372813689 & 0.003350412 \\
\hline MNXI-ASI & Oxaliplatin & 0.360297862 & 0.004686991 \\
\hline MNXI-ASI & Nelfinavir & 0.341952173 & 0.007491512 \\
\hline MNXI-ASI & Nitrogen mustard & 0.337752405 & 0.008309364 \\
\hline MNXI-ASI & Belinostat & 0.335705073 & 0.008735515 \\
\hline MNXI-ASI & NMS-E628 & 0.328447379 & 0.010403107 \\
\hline MNXI-ASI & Imiquimod & $0.32648888 I$ & 0.010897833 \\
\hline MNXI-ASI & Isotretinoin & 0.311272205 & 0.015483899 \\
\hline MNXI-ASI & Ixabepilone & 0.310681462 & 0.015691099 \\
\hline MNXI-ASI & Dexrazoxane & 0.30700077 & 0.017036333 \\
\hline MNXI-ASI & Alectinib & 0.295927495 & 0.021692672 \\
\hline MNXI-ASI & METHOTREXATE & 0.290846029 & 0.024166521 \\
\hline MNXI-ASI & Tamoxifen & 0.285979157 & 0.026755117 \\
\hline MNXI-ASI & Gilteritinib & 0.279007524 & 0.030866456 \\
\hline MNXI-ASI & Brigatinib & 0.259510007 & 0.045250555 \\
\hline MNXI-ASI & Palbociclib & $0.2585543 \mid 5$ & 0.046077575 \\
\hline MNXI-ASI & Raloxifene & 0.254734547 & 0.049507747 \\
\hline ZFASI & Everolimus & -0.436663069 & 0.000486505 \\
\hline ZFASI & Palbociclib & 0.430953581 & 0.000587332 \\
\hline ZFASI & RAPAMYCIN & -0.346415899 & 0.006700172 \\
\hline ZFASI & Rapamycin & -0.325746469 & 0.011090622 \\
\hline ZFASI & LEE-0I I & 0.323890808 & 0.011585449 \\
\hline ZFASI & Dexrazoxane & 0.301780769 & 0.019112373 \\
\hline ZFASI & Abiraterone & -0.299755958 & 0.019973715 \\
\hline ZFASI & Temsirolimus & -0.293168167 & 0.023007955 \\
\hline ZFASI & Ifosfamide & 0.282430683 & 0.028786168 \\
\hline ZFASI & Hydroxyurea & 0.266073929 & 0.039893835 \\
\hline
\end{tabular}




\section{Abbreviations}

PRCC, Papillary renal cell carcinoma; IncRNA, Long noncoding RNA; TCGA, The Cancer Genome Atlas; ROC, Receiver Operating Characteristic; AUC, Area under the curve; OSR, Overall survival rate; K-M, Kaplan-Meier; GSEA, Gene set enrichment analysis; GO, Gene Ontology; KEGG, Kyoto Encyclopedia of Genes and Genomes.

\section{Data Sharing Statement}

The original contributions presented in this study are included in the article/supplementary material, and further inquiries can be directed to the corresponding authors.

\section{Ethical Statement}

The Ethics Committee of Beijing Chaoyang Hospital has waived the need for ethical approval, and the reasoning why the ethics approval has been waived.

\section{Acknowledgments}

This study is supported by Beijing Municipal Natural Science Foundation (Grant No. 7204308), The National Natural Science Foundation of China (Grant No. 81900605), The National Natural Science Foundation of China (Grant No. 81901404), and Beijing Hospitals Authority Youth Program (No. QML20210303).

\section{Author Contributions}

All authors made a significant contribution to the work reported, whether that is in the conception, study design, execution, acquisition of data, analysis and interpretation, or in all these areas; took part in drafting, revising or critically reviewing the article; gave final approval of the version to be published; have agreed on the journal to which the article has been submitted; and agree to be accountable for all aspects of the work.

\section{Disclosure}

The authors report no conflicts of interest in this work.

\section{References}

1. Sung H, Ferlay J, Siegel RL, et al. Global cancer statistics 2020: GLOBOCAN estimates of incidence and mortality worldwide for 36 cancers in 185 countries. CA Cancer J Clin. 2021;71(3):209-249. doi: $10.3322 /$ caac. 21660

2. Rhoades Smith KE, Bilen MA. A review of papillary renal cell carcinoma and MET inhibitors. Kidney Cancer. 2019;3(3):151-161. doi:10.3233/KCA-190058
3. Alomari AK, Nettey OS, Singh D, Kluger H, Adeniran AJ. Clinicopathological and immunohistochemical characteristics of papillary renal cell carcinoma with emphasis on subtyping. Hum Pathol. 2015;46(10):1418-1426. doi:10.1016/j.humpath.2015.06.006

4. Akhtar M, Al-Bozom IA, Al Hussain T. Papillary Renal Cell Carcinoma (PRCC): an update. Adv Anat Pathol. 2019;26 (2):124-132. doi:10.1097/PAP.0000000000000220

5. Mou Y, Wang J, Wu J, et al. Ferroptosis, a new form of cell death: opportunities and challenges in cancer. J Hematol Oncol. 2019;12 (1):34. doi:10.1186/s13045-019-0720-y

6. Latunde-Dada GO. Ferroptosis: role of lipid peroxidation, iron and ferritinophagy. Biochim Biophys Acta Gen Subj. 2017;1861 (8):1893-1900. doi:10.1016/j.bbagen.2017.05.019

7. Hirschhorn T, Stockwell BR. The development of the concept of ferroptosis. Free Radic Biol Med. 2019;133:130-143. doi:10.1016/j. freeradbiomed.2018.09.043

8. Chandra Gupta S, Nandan Tripathi Y. Potential of long non-coding RNAs in cancer patients: from biomarkers to therapeutic targets. Int J Cancer. 2017;140(9):1955-1967. doi:10.1002/ijc.30546

9. Zhang X, Wang W, Zhu W, et al. Mechanisms and functions of long non-coding RNAs at multiple regulatory levels. Int $J$ Mol Sci. 2019;20(22):22. doi:10.3390/ijms20225573

10. Wang C, Kawakami-Mori F, Kang L, et al. Low-dose L-NAME induces salt sensitivity associated with sustained increased blood volume and sodium-chloride cotransporter activity in rodents. Kidney Int. 2020;98(5):1242-1252. doi:10.1016/j.kint.20 20.05.050

11. Chi Y, Wang J, Wang J, Yu W, Yang J. Long non-coding RNA in the pathogenesis of cancers. Cells. 2019;8(9):1015. doi:10.3390/ cells8091015

12. Yao RW, Wang Y, Chen LL. Cellular functions of long noncoding RNAs. Nat Cell Biol. 2019;21(5):542-551. doi:10.1038/s41556-0190311-8

13. $\mathrm{Lu} \mathrm{J}, \mathrm{Xu} \mathrm{F}, \mathrm{Lu} \mathrm{H}$. LncRNA PVT1 regulates ferroptosis through miR-214-mediated TFR1 and p53. Life Sci. 2020;260:118305. doi:10.1016/j.lfs.2020.118305

14. Wang M, Mao C, Ouyang L, et al. Long noncoding RNA LINC00336 inhibits ferroptosis in lung cancer by functioning as a competing endogenous RNA. Cell Death Differ. 2019;26(11):2329-2343. doi:10.1038/s41418-019-0304-y

15. Wang Z, Jensen MA, Zenklusen JC. A practical guide to The Cancer Genome Atlas (TCGA). Methods Mol Biol. 2016;1418:111-141.

16. Zhou N, Bao J. FerrDb: a manually curated resource for regulators and markers of ferroptosis and ferroptosis-disease associations. Database (Oxford). 2020;2020. doi:10.1093/database/baaa021

17. Yu G, Wang LG, Han Y, He QY. clusterProfiler: an R package for comparing biological themes among gene clusters. OMICS. 2012;16 (5):284-287. doi:10.1089/omi.2011.0118

18. Kanehisa M, Sato Y, Kawashima M. KEGG mapping tools for uncovering hidden features in biological data. Protein Sci. 2021. doi:10.1002/pro.4172

19. Doncheva NT, Morris JH, Gorodkin J, Jensen LJ. Cytoscape StringApp: network analysis and visualization of proteomics data. $J$ Proteome Res. 2019;18(2):623-632. doi:10.1021/acs. jproteome.8b00702

20. Subramanian A, Tamayo P, Mootha VK, et al. Gene set enrichment analysis: a knowledge-based approach for interpreting genome-wide expression profiles. Proc Natl Acad Sci USA. 2005;102 (43):15545-15550. doi:10.1073/pnas.0506580102

21. Charoentong P, Finotello F, Angelova M, et al. Pan-cancer immunogenomic analyses reveal genotype-immunophenotype relationships and predictors of response to checkpoint blockade. Cell Rep. 2017;18(1):248-262. doi:10.1016/j.celrep.2016.12.019

22. Yoshihara K, Shahmoradgoli M, Martinez E, et al. Inferring tumour purity and stromal and immune cell admixture from expression data. Nat Commun. 2013;4:2612. doi:10.1038/ncomms3612 
23. Shi J, Jiang D, Yang S, et al. LPAR1, correlated with immune infiltrates, is a potential prognostic biomarker in prostate cancer. Front Oncol. 2020;10:846. doi:10.3389/fonc.2020.00846

24. Yi M, Nissley DV, McCormick F, Stephens RM. ssGSEA score-based Ras dependency indexes derived from gene expression data reveal potential Ras addiction mechanisms with possible clinical implications. Sci Rep. 2020;10(1):10258. doi:10.1038/s41598-02066986-8

25. Li T, Fan J, Wang B, et al. TIMER: a web server for comprehensive analysis of tumor-infiltrating immune cells. Cancer Res. 2017;77(21): e108-e110. doi:10.1158/0008-5472.CAN-17-0307

26. Luo L, Zhou H, Su H. Identification of 4-genes model in papillary renal cell tumor microenvironment based on comprehensive analysis. BMC Cancer. 2021;21(1):553. doi:10.1186/s12885-021-08319-0

27. Liu Z, Wan Y, Yang M, et al. Identification of methylation-driven genes related to the prognosis of papillary renal cell carcinoma: a study based on The Cancer Genome Atlas. Cancer Cell Int. 2020;20:235. doi:10.1186/s12935-020-01331-7

28. O'Brien SJ, Fiechter C, Burton J, et al. Long non-coding RNA ZFAS1 is a major regulator of epithelial-mesenchymal transition through miR-200/ZEB1/E-cadherin, vimentin signaling in colon adenocarcinoma. Cell Death Discov. 2021;7(1):61. doi:10.1038/ s41420-021-00427-x

29. Chen SH, Lin F, Zhu JM, et al. An immune-related IncRNA prognostic model in papillary renal cell carcinoma: a lncRNA expression analysis. Genomics. 2021;113(1 Pt 2):531-540. doi:10.1016/j. ygeno.2020.09.046

30. Cheng G, Liu D, Liang H, Yang H, Chen K, Zhang X. A cluster of long non-coding RNAs exhibit diagnostic and prognostic values in renal cell carcinoma. Aging (Albany NY). 2019;11(21):9597-9615. doi:10.18632/aging.102407

31. Chen K, Gan JX, Huang ZP, Liu J, Liu HP. Clinical significance of long noncoding RNA MNX1-AS1 in human cancers: a meta-analysis of cohort studies and bioinformatics analysis based on TCGA datasets. Bioengineered. 2021;12(1):875-885. doi:10.1080/ 21655979.2021.1888596

32. Zhao J, Song X, Xu T, et al. Identification of potential prognostic competing triplets in high-grade serous ovarian cancer. Front Genet. 2020;11:607722. doi:10.3389/fgene.2020.607722
33. Gong S, Xu M, Zhang Y, Shan Y, Zhang H. The prognostic signature and potential target genes of six long non-coding RNA in laryngeal squamous cell carcinoma. Front Genet. 2020;11:413. doi:10.3389/ fgene. 2020.00413

34. Zuo S, Wang L, Wen Y, Dai G. Identification of a universal 6-IncRNA prognostic signature for three pathologic subtypes of renal cell carcinoma. J Cell Biochem. 2018;120:7375.

35. Yang F, Song Y, Ge L, Zhao G, Liu C, Ma L. Long non-coding RNAs as prognostic biomarkers in papillary renal cell carcinoma. Oncol Lett. 2019;18(4):3691-3697. doi:10.3892/ol.2019.10684

36. Wen D, Huang Z, Li Z, et al. LINC02535 co-functions with PCBP2 to regulate DNA damage repair in cervical cancer by stabilizing RRM1 mRNA. J Cell Physiol. 2020;235(10):7592-7603. doi:10.1002/jcp.29667

37. Li Y, Shen R, Wang A, et al. Construction of a prognostic immune-related LncRNA risk model for lung adenocarcinoma. Front Cell Dev Biol. 2021;9:648806. doi:10.3389/fcell.2021.648806

38. Fang S, Zhao Y, Hu X. LncRNA ADAMTS9-AS1 Restrains the aggressive traits of breast carcinoma cells via sponging miR-513a5p. Cancer Manag Res. 2020;12:10693-10703. doi:10.2147/CMAR. S266575

39. Chen W, Tu Q, Yu L, et al. LncRNA ADAMTS9-AS1, as prognostic marker, promotes cell proliferation and EMT in colorectal cancer. Hum Cell. 2020;33(4):1133-1141. doi:10.1007/s13577-020-00388-w

40. Bai $\mathrm{Y}$, Lin $\mathrm{H}$, Chen $\mathrm{J}, \mathrm{Wu} \mathrm{Y,} \mathrm{Yu} \mathrm{S}$. Identification of prognostic glycolysis-related lncRNA signature in tumor immune microenvironment of Hepatocellular carcinoma. Front Mol Biosci. 2021;8:645084. doi:10.3389/fmolb.2021.645084

41. Wang S, Chen X, Qiao T. Long noncoding RNA MIR44352HG promotes the progression of head and neck squamous cell carcinoma by regulating the miR3835p/RBM3 axis. Oncol Rep. 2021;45(6). doi:10.3892/or.2021.8050

42. Xing P, Wang Y, Zhang L, Ma C, Lu J. Knockdown of lncRNA MIR44352HG and ST8SIA1 expression inhibits the proliferation, invasion and migration of prostate cancer cells in vitro and in vivo by blocking the activation of the FAK/AKT/betacatenin signaling pathway. Int J Mol Med. 2021;47(6). doi:10.3892/ijmm.2021.4926
International Journal of General Medicine

\section{Publish your work in this journal}

The International Journal of General Medicine is an international, peer-reviewed open-access journal that focuses on general and internal medicine, pathogenesis, epidemiology, diagnosis, monitoring and treatment protocols. The journal is characterized by the rapid reporting of reviews, original research and clinical studies across all disease areas. The manuscript management system is completely online and includes a very quick and fair peer-review system, which is all easy to use. Visit http://www.dovepress.com/ testimonials.php to read real quotes from published authors. 